\title{
Iris Recognition using Self Mutated Hybrid Wavelet Transform using Cosine, Haar, Hartley and Slant Transforms with Partial Energies of Transformed Iris Images
}

\author{
Tejas H. Jadhav \\ Department of Information Technology \\ Pimpri Chinchwad College of Engineering \\ Pune, India
}

\author{
Jaya $H$. Dewan \\ Department of Information Technology \\ Pimpri Chinchwad College of Engineering \\ Pune, India
}

\begin{abstract}
The paper presents iris recognition technique based on the concept of Energy Compaction. Compaction is done by using the partial energies of transformed iris images. Various Self mutated hybrid wavelet transforms (SMHWT) namely 'Cosine-Haar', 'Cosine-Hartley', and 'Cosine-Slant' are used to generate feature vector of iris images. The important task is reducing the size of feature vector so that the performance of system can be increased. The reduction of size of feature vector is achieved by using the concept of partial energies. The size of feature vector reduces immensely for $99 \%, 98 \%$, $97 \%$ and $96 \%$ of energy. The size of feature vector is extremely large while considering all the coefficients of transformed iris images for $100 \%$ of energy which leads more computation. System gives better performance when partial energies are considered. The proposed technique is tested on Palacky University Iris Database. Genuine Acceptance Rate (GAR) is used as a metric to test the performance of the proposed Iris recognition technique. The self mutated hybrid wavelet transform of 'Cosine-Haar' gives preeminent GAR value as compared to other transforms considered. Results show the proposed technique gives better performance in terms of accuracy by considering partial energies as compared to $100 \%$ energy.
\end{abstract}

\section{General Terms}

Iris Recognition, Feature Extraction.

\section{Keywords}

Iris Recognition, Feature Vector, Genuine Acceptance Rate, Energy Compaction, Self Mutated Hybrid Wavelet Transform, Cosine transform, Haar Transform, Hartley Transform, Slant Transform.

\section{INTRODUCTION}

Iris recognition is an automated method of biometric identification that uses mathematical pattern-recognition techniques on images of the irides of an individual's eyes whose complex random patterns are unique $[3,5,7]$.

The spatial patterns that are present in the human iris are highly distinctive to an individual. Unlike the human face, however, the difference in appearance of any one iris might be enough unique to make possible an automated recognition system based on currently available machine vision technologies.

The main modules of Iris Recognition techniques are preprocessing, feature extraction and matching. Feature extraction is most important task in iris recognition techniques as features are extracted from each eye image and feature vector is generated [1,8]. Various combinations of Self Mutated Hybrid Wavelet transforms are used to generate the transformed iris images. From these transformed images, feature vector is generated by using the concept of partial energies. In feature extraction module, feature vector is generated by considering all the coefficients of transformed iris image i.e. $100 \%$ of energy. The remaining ways uses the number of coefficients that hold $99 \%, 98 \%, 97 \%$ and $96 \%$ of the energies for generating the feature vector. The size of feature vector is dependent on the number of coefficients considered. The size of feature vector is extremely large when all the coefficients of transformed iris image are considered thus, the computations are also more. But when the partial energies are considered to generate the feature vector, the numbers of coefficients required are very less. So, the size of feature vector reduces immensely and thus, the numbers of computations are also less. So, the speed of system increases without compromising the performance.

The first step of feature extraction module is to find out the number of coefficients to be considered for expected Energy Percentages. This can be obtained by the concept of Energy Compaction which is elaborated in further sections.

\section{SELF MUTATED HYBRID WAVELET TRANSFORM}

The Hybrid Wavelet transform matrix $\mathrm{H}_{\mathrm{AB}}$ can be generated from two orthogonal transform matrices (say $\mathrm{A}$ and $\mathrm{B}$ respectively with sizes $\mathrm{P} \times \mathrm{P}$ and $\mathrm{Q} \times \mathrm{Q}$, where size of $\mathrm{H}_{\mathrm{AB}}$ is $\mathrm{PQ} \times \mathrm{PQ})[2,6]$. The generation of Hybrid Wavelet transform is shown by Fig.1. Here first $Q$ numbers of rows of the hybrid wavelet transform matrix are calculated as the Kronecker product of each element of first row of the orthogonal transform matrix A with each of the columns of the orthogonal transform matrix B. Further for next Q number of rows of hybrid wavelet transform matrix, the second row of the orthogonal transform matrix A is shift rotated after being appended with zeros. Similarly, the other rows of hybrid wavelet transform matrix are generated.

The self mutated hybrid wavelet transform matrix SH of size $\mathrm{N} \times \mathrm{N}$ can be generated from two hybrid wavelet transform matrices \{ say $\mathrm{H}_{\mathrm{AB}}$ and $\mathrm{H}_{\mathrm{XY}}$ with sizes $\mathrm{PQ} \times \mathrm{PQ}$ and $\mathrm{ST} \times \mathrm{ST}$ respectively $\}$. 


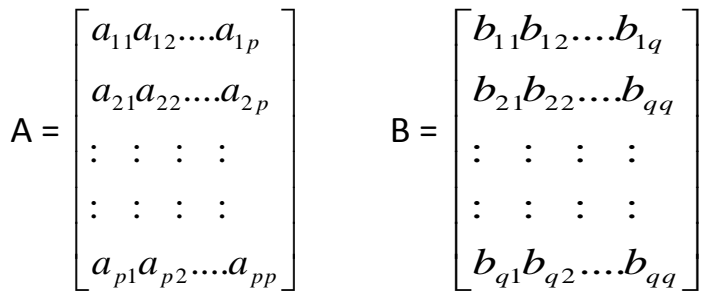

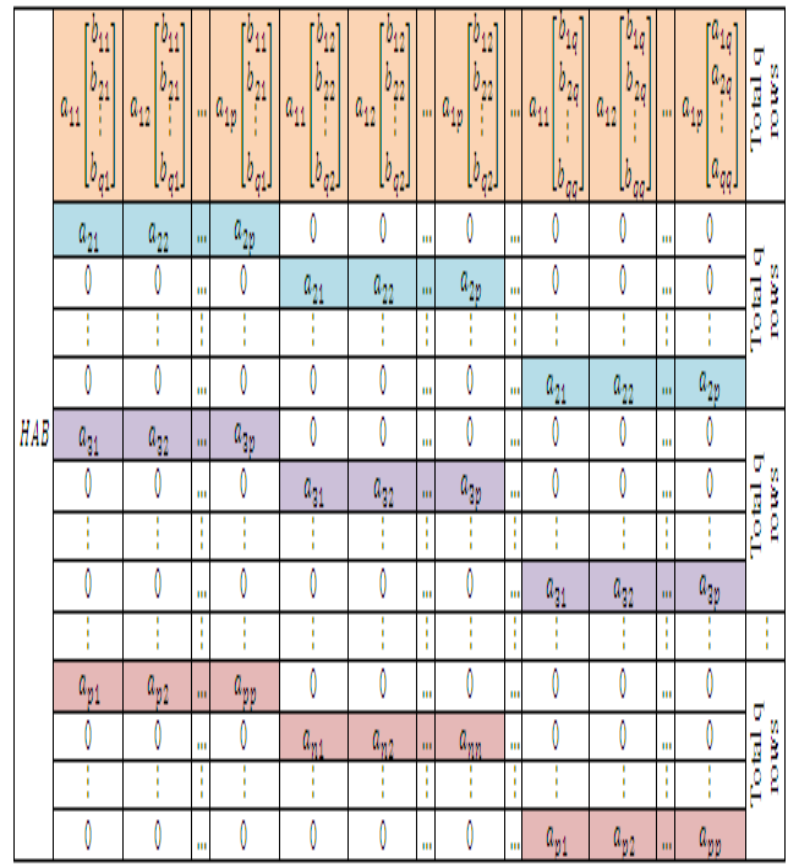

Fig 1: Generation of Hybrid Wavelet transform

\section{PROPOSED IRIS RECOGNITION TECHNIQUE}

In proposed iris recognition technique, self mutated hybrid wavelet transforms matrix of size $256 \times 256$ is generated by combining two hybrid wavelet transform matrices of size $16 \times 16$ which are generated by combining Cosine transform matrix of size $4 \times 4$ with three different orthogonal transform matrices of size $4 \times 4$ namely Haar, Hartley and Slant transform $[1,4,9]$. The Self Mutated Hybrid Wavelet transform matrix is generated and then applied on the input iris images to get transformed iris images. From such transformed iris images, feature vector is generated. For $100 \%$ energy, all coefficients of transformed iris images are considered to generate the feature vector and hence the size of feature vector is extremely large. But when the partial energies are considered, the coefficients holding the partial energies are only considered to generate the feature vector thus, the number of coefficients required are less and the size of feature vector reduces immensely. For the particular energy percentage, the number of coefficients that needs to be considered to form the feature vector is calculated using the concept of energy compaction.

When a transform is applied on an image, it desegregates the low frequency and the high frequency components from each other. The low frequency components have more information about the image as compared to the high frequency components. As, these low frequency coefficients have more details of the image, they can be used to generate the feature vector. This property of transform is called energy compaction. By using this property of transforms, the size of feature vector can be reduced. The steps for energy compaction are depicted in fig. 2.

Here, Palacky University Dataset contains 384 images are used as test bed for experimentation. For a particular energy percentage the number of coefficients that needs to be considered to generate feature vector is represented by Coefficient table. Coefficient table is found out by carrying out steps shown in fig. 2. Self mutated hybrid wavelet transform is applied on all 384 iris images to get 384 transformed iris images. Result matrix is generated by taking element wise addition of energies of all the 384 transformed iris images. Next, each element of result matrix is divided by 384 to generate average energy matrix. Average energy matrix represents the average energy compaction done by the transform on all the images in database. Next the average energy matrix is stored as a four column matrix with first column representing the coefficient value, second column representing the squared coefficient value, third and fourth column representing the row and column index. The above generated matrix is then sorted by second column in descending order and the corresponding column values are swapped to get final average energy matrix. From the final average energy matrix the energy values are added from the first coefficient to the last coefficient in a cumulative way to obtain Summed Energy matrix. Summed energy matrix represents the summed energy up to the considered coefficient. Summed energy matrix is a two column matrix with first column representing the number of coefficients and second column representing the energy values. From the summed energy matrix coefficient table is generated by simply scanning the Summed Energy matrix for a particular energy value and the corresponding column value represents the number of coefficients that needs to be considered for generating feature vector for that particular energy value.

In this way coefficient table for all the self mutated hybrid wavelet transforms can be found out. As the percentage of energy considered is reduced, the number of coefficients required also reduces immensely.

\subsection{Feature Extraction}

Following steps show the creation of a feature vector dataset for $99 \%$ of partial energy.

1. Read $\mathrm{N} \times \mathrm{N} \times 3$ iris image.

2. Resize it to $256 \times 256 \times 3$.

3. Separate into R, G and B planes.

4. Apply Self Mutated Hybrid Wavelet transform 'SH' to get transformed iris images.

5. Generate feature vector from these transformed iris images by considering the number of coefficients for $99 \%$ energy by following the concept of Energy compaction. 


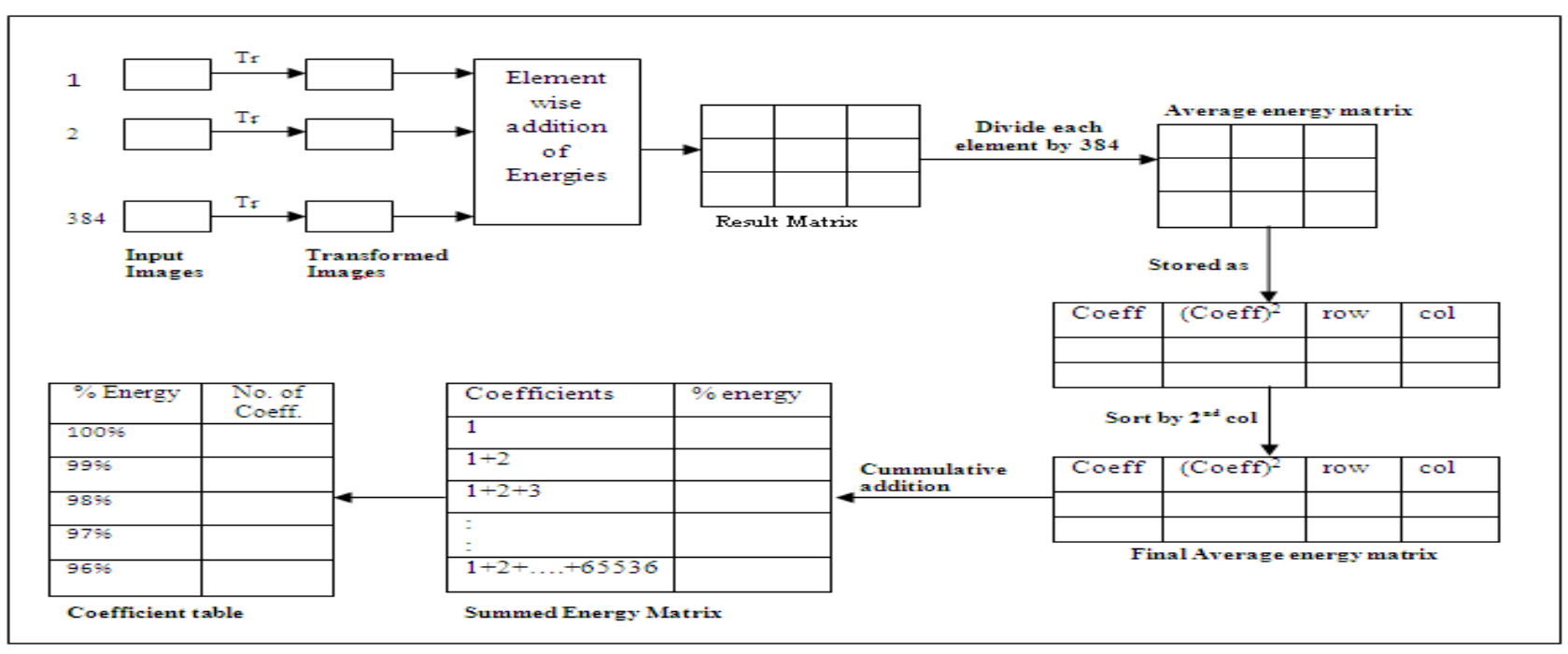

Fig 2: Energy Compaction

\subsection{Query Execution}

Query iris image is taken as an input image. Following steps are done in query execution module.

1. Read input query image.

2. Resize it to $256 \times 256 \times 3$.

3. Separate into $R, G$ and $B$ planes.

4. Apply Self Mutated Hybrid Wavelet transform to get transformed query iris images.

5. From these transformed query images generate query feature vector by considering the number of coefficients for corresponding partial energy.

6. Next matching is done between the query feature vector and all the feature vectors in the feature vector database using Mean Square Error (MSE) similarity measure to find the first six best relevant matches from the database.

7. Based on the person for whom maximum numbers of best relevant matches are found in step 6, the decision about recognition of query image is done.

\section{IMPLEMENTATION}

\subsection{Test Bed}

The implementation is done on Matlab 2012a, Intel core 3 with 4 GB RAM. Proposed technique is tested on Palacky University Database which consists of 384 iris images. Database contains 64 persons images of both males and females. Per person six images are taken, in which 3 for left eye and 3 for right eye [10]. Sample input images from the database are shown in Fig. 3.

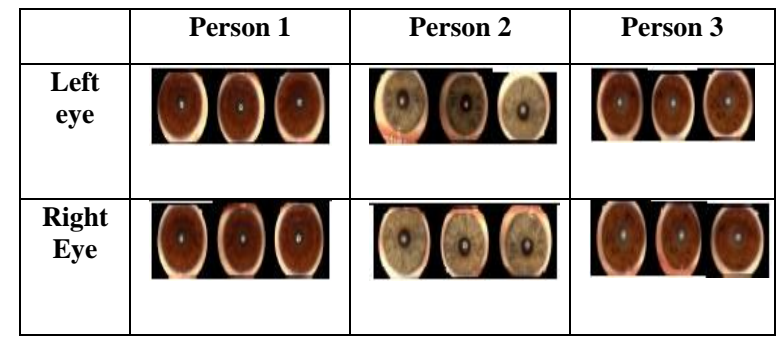

Fig 3: Sample Images from Palacky University Iris Database

\subsection{Similarity Measurement Criteria}

Mean square error (MSE) is used as similarity measurement criteria. MSE between two feature vectors $\mathrm{x}$ and $\mathrm{y}$ is calculated as follows,

$$
M S E=1 / N \sum_{i=1}^{N}\left(x_{i}-y_{i}\right)^{2}
$$

Where, $\mathrm{N}$ is the size of the vectors to be compared. Lower the MSE, higher is the similarity between the feature vectors $\mathrm{x}$ and $\mathrm{y}$.

\subsection{Performance Comparison Technique}

Genuine Acceptance Rate (GAR) is used as a performance comparison metric to evaluate the performance of proposed iris recognition system GAR is defined by equation (2).

$$
\mathrm{GAR}=\frac{\text { Number of Correct Acceptence s }}{\text { Number of Identifica tion Attempts }}
$$

\section{RESULTS AND DISCUSSIONS}

Table 1 shows the performances given by Self Mutated Hybrid Wavelet transforms of Cosine-Haar, Cosine-Hartley and Cosine-Slant for $100 \%, 99 \%, 98 \%, 97 \%$ and $96 \%$ of partial energies. Also it shows that the performances given by the Hybrid Wavelet transforms (HWT) of the Cosine-Haar, Cosine-Hartley and Cosine-Slant transforms for Partial energies. It shows that Self Mutated Hybrid Wavelet transform of Cosine-Haar gives highest GAR of $81 \%$ for $99 \%$ energy. By considering partial energies the size of feature vector reduced immensely and performance is increased. For all Self Mutated Hybrid Wavelet transform using 100\% energy give GAR of $67 \%$. Best Performance is given by Cosine-Haar Self Mutated Hybrid Wavelet transform with GAR of $81 \%$ using $99 \%$ and $98 \%$ of energy with $14 \%$ improvement in GAR. Results show that Hybrid Wavelet transform of Cosine-Haar gives GAR of $80 \%$ for $99 \%$ of energy and GAR of $81 \%$ for $98 \%$ of energy.

For Self Mutated Hybrid Wavelet transform of Cosine-Haar the number of coefficients considered to form feature vector is 20 for $99 \%$ of energy and the number of coefficients considered to form feature vector is 11 for $98 \%$ of energy. The percentage reduction in size of feature vector for $98 \%$ of energy is $98.32 \%$ while the percentage reduction in size of feature vector for $99 \%$ of energy is $96.94 \%$. Second best 
performance is given by Cosine-Haar Self Mutated Hybrid Wavelet transform with GAR of $80 \%$ using $97 \%$ and $96 \%$ of energy with $13 \%$ improvement in GAR. The Self Mutated Hybrid Wavelet transform of Cosine-Hartley gives GAR of $71 \%$ for $99 \%$ of energy. The Hybrid Wavelet transform of Cosine-Hartley gives GAR of 70\% for $99 \%$ of energy.

Table 1. Summary of performance given by Self Mutated Hybrid Wavelet transforms for different energies

\begin{tabular}{|c|c|c|c|c|c|}
\hline $\begin{array}{c}\text { Transf } \\
\text { orms }\end{array}$ & $\begin{array}{c}\text { Ener } \\
\text { gy } \\
\text { consi } \\
\text { dere } \\
\text { d to } \\
\text { form } \\
\text { featu } \\
\text { re } \\
\text { vecto } \\
\text { r }\end{array}$ & $\begin{array}{c}\begin{array}{c}\text { Number } \\
\text { of } \\
\text { coefficie }\end{array} \\
\text { nts } \\
\text { consider } \\
\text { ed to } \\
\text { form } \\
\text { feature } \\
\text { vector }\end{array}$ & $\begin{array}{l}\text { Percent } \\
\text { age } \\
\text { Reduct } \\
\text { ion in } \\
\text { size of } \\
\text { feature } \\
\text { vector }\end{array}$ & $\overline{\text { GAR }}$ & $\begin{array}{c}\text { Perce } \\
\text { ntage } \\
\text { impro } \\
\text { veme } \\
\text { nt in } \\
\text { GAR }\end{array}$ \\
\hline \multirow{5}{*}{$\begin{array}{c}\text { SMHW } \\
\text { T using } \\
\text { Cosin- } \\
\text { Haar } \\
\text { transfor } \\
\text { m }\end{array}$} & $100 \%$ & 65536 & 0 & $67 \%$ & -- \\
\hline & $99 \%$ & 20 & $96.94 \%$ & $81 \%$ & $14 \%$ \\
\hline & $98 \%$ & 11 & $98.32 \%$ & $81 \%$ & $14 \%$ \\
\hline & $97 \%$ & 8 & $98.77 \%$ & $80 \%$ & $13 \%$ \\
\hline & $96 \%$ & 8 & $98.77 \%$ & $80 \%$ & $13 \%$ \\
\hline \multirow{5}{*}{$\begin{array}{c}\text { HWT } \\
\text { using } \\
\text { Cosine- } \\
\text { Haar } \\
\text { transfor } \\
\text { m }\end{array}$} & $100 \%$ & 65536 & 0 & $67 \%$ & -- \\
\hline & $99 \%$ & 20 & $96.94 \%$ & $80 \%$ & $13 \%$ \\
\hline & $98 \%$ & 11 & $98.32 \%$ & $81 \%$ & $14 \%$ \\
\hline & $97 \%$ & 7 & $98.93 \%$ & $79 \%$ & $12 \%$ \\
\hline & $96 \%$ & 7 & $98.93 \%$ & $79 \%$ & $12 \%$ \\
\hline \multirow{5}{*}{$\begin{array}{c}\text { SMHW } \\
\text { T using } \\
\text { Cosine- } \\
\text { Hartley } \\
\text { transfor } \\
\text { m }\end{array}$} & $100 \%$ & 65536 & 0 & $67 \%$ & -- \\
\hline & $99 \%$ & 11 & $98.32 \%$ & $71 \%$ & $4 \%$ \\
\hline & $98 \%$ & 8 & $98.77 \%$ & $70 \%$ & $3 \%$ \\
\hline & $97 \%$ & 5 & $99.23 \%$ & $68 \%$ & $1 \%$ \\
\hline & $96 \%$ & 4 & $99.38 \%$ & $68 \%$ & $1 \%$ \\
\hline \multirow{5}{*}{$\begin{array}{c}\text { HWT } \\
\text { using } \\
\text { Cosine- } \\
\text { Hartley } \\
\text { transfor } \\
\text { m }\end{array}$} & $100 \%$ & 65536 & 0 & $67 \%$ & -- \\
\hline & $99 \%$ & 11 & $98.32 \%$ & $70 \%$ & $3 \%$ \\
\hline & $98 \%$ & 7 & $98.93 \%$ & $69 \%$ & $2 \%$ \\
\hline & $97 \%$ & 5 & $99.23 \%$ & $67 \%$ & $0 \%$ \\
\hline & $96 \%$ & 5 & $99.23 \%$ & $67 \%$ & $0 \%$ \\
\hline \multirow{5}{*}{$\begin{array}{c}\text { SMHW } \\
\text { T using } \\
\text { Cosine- } \\
\text { Slant } \\
\text { transfor } \\
\text { m }\end{array}$} & $100 \%$ & 65536 & 0 & $67 \%$ & -- \\
\hline & $99 \%$ & 26 & $96.03 \%$ & $70 \%$ & $3 \%$ \\
\hline & $98 \%$ & 17 & $97.40 \%$ & $69 \%$ & $2 \%$ \\
\hline & $97 \%$ & 13 & $98.01 \%$ & $67 \%$ & $0 \%$ \\
\hline & $96 \%$ & 11 & $98.32 \%$ & $67 \%$ & $0 \%$ \\
\hline \multirow{5}{*}{$\begin{array}{c}\text { HWT } \\
\text { using } \\
\text { Cosine- } \\
\text { Slant } \\
\text { transfor } \\
\text { m }\end{array}$} & $100 \%$ & 65536 & 0 & $67 \%$ & -- \\
\hline & $99 \%$ & 27 & $95.88 \%$ & $69 \%$ & $2 \%$ \\
\hline & $98 \%$ & 18 & $98.72 \%$ & $68 \%$ & $1 \%$ \\
\hline & $97 \%$ & 13 & $98.01 \%$ & $67 \%$ & $0 \%$ \\
\hline & $96 \%$ & 13 & $98.01 \%$ & $67 \%$ & $0 \%$ \\
\hline
\end{tabular}

Fig. 4 shows the GAR values for different Self Mutated Hybrid Wavelet transforms and energies. Considering Partial energies gives better GAR value than by considering $100 \%$ of energy and also it reduces the size of feature vector immensely. The Cosine-Haar Self Mutated Hybrid Wavelet transform (SMHWT) is giving better GAR as compared to Self Mutated Hybrid Wavelet transform of Cosine-Hartley and Cosine-Slant for all energy percentages. For $99 \%$ and $98 \%$ of partial energies GAR of SMHWT of Cosine-Haar is $81 \%$. For $97 \%$ and $96 \%$ of partial energies GAR of SMHWT of Cosine-Haar is $80 \%$.

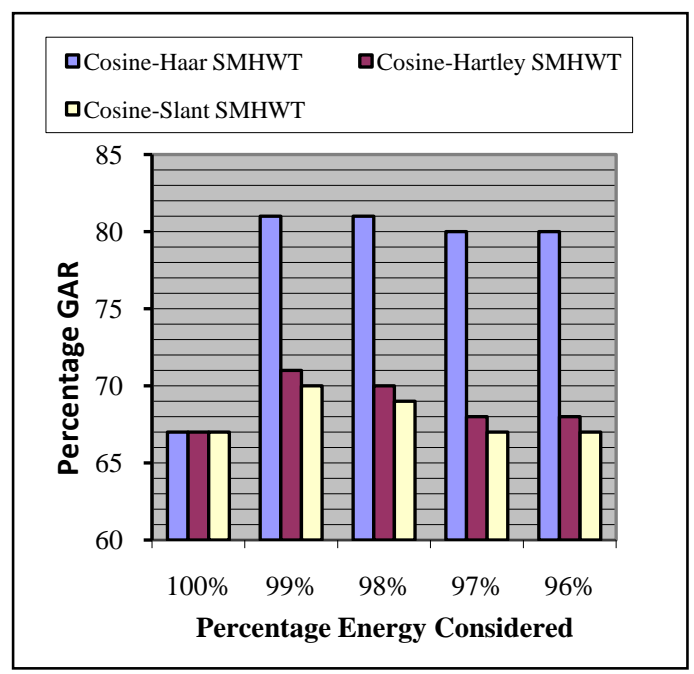

Fig 4: Performance Comparison of different percentages of energies for respective Self Mutated Hybrid Wavelet Transforms

Fig. 5 shows the GAR values for different percentages of energies and hybrid wavelet transforms (HWT) for proposed iris recognition techniques generated using Cosine-Haar, Cosine-Hartley and Cosine-Slant transform. Results show that the hybrid wavelet transform generated using Cosine-Haar gives better GAR as compared to all other hybrid wavelet transform considered.

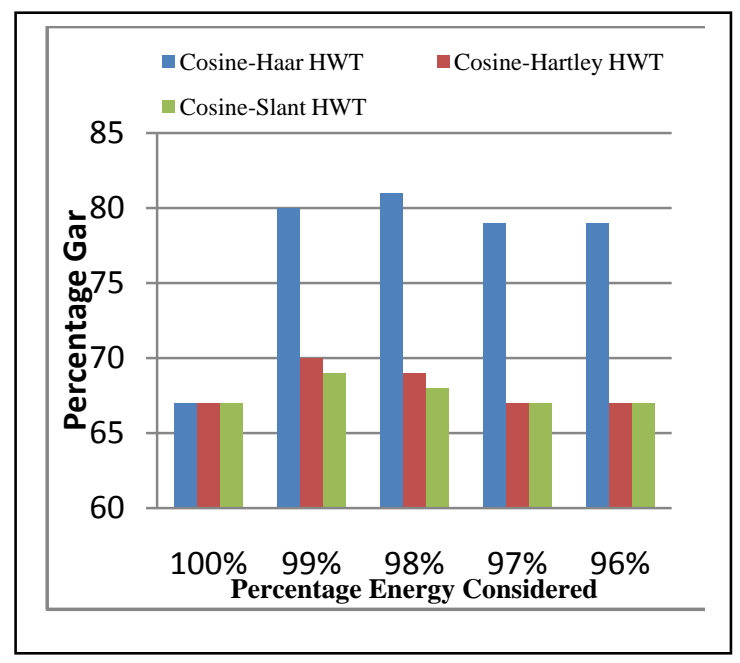

Fig 5: Performance Comparison of Hybrid Wavelet transforms for respective percentage of partial energies.

\section{CONCLUSION}

Here, the iris recognition technique is proposed using self mutated hybrid wavelet transforms. The SMHWT reduces the size of feature vector immensely and gives better performance. Results show that among all the self mutated hybrid wavelet transforms, Cosine-Haar self mutated hybrid wavelet transform gives GAR of $81 \%$ using $99 \%$ and $98 \%$ of partial energy with $14 \%$ improvement in GAR. The task of reducing the size of feature vector is achieved by using the concept of energy compaction and partial energies without affecting the performance of the system. Accuracy of the system is improved by considering the partial energies are improved as compared to considering $100 \%$ of energy. Energy compaction 
and self mutated hybrid wavelet transforms helped in reducing the feature vector size to the extent of $96.03 \%$ to $99.38 \%$. In future, the colour spaces will be used to test the effect on the performance of system.

\section{REFERENCES}

[1] Dr. Sudeep Thepade, Pushpa R. Mandal ,"Energy Compaction based Novel Iris Recognition Techniques using Partial Energies of Transformed Iris Images with Cosine, Walsh, Haar, Kekre, Hartley Transforms and their Wavelet Transforms",Annual IEEE India Conference (INDICON),2014.

[2] Dr.Sudeep Thepade, Jaya H. Dewan, "Image Compression using Cosine - Slant Hybrid Wavelet Transform with Assorted Color Spaces", International Journal of Computer Applications (0975 - 8887) Volume 73- No.7, July 2013.

[3] Dr. Sudeep D. Thepade, Pooja Bidwai, "Iris Recognition using Fractional Coefficients of Transforms, Wavelet Transforms and Hybrid Wavelet Transforms", International Conference on Control, Computing, Communication and Materials (ICCCCM), 2011.

[4] Ameya Deshpande, Sumitkumar Dubey, Hrushikesh Shaligram, Aditya Potnis, Satishkumar Chavan,"Iris Recognition System using Block Based Approach with DWT and DCT", Annual IEEE India Conference (INDICON), 2014

[5] Swati Dhage, Sushma Hegde, Manikantan K, S. Ramchandram, " DWT based feature extraction and Randon transform based contrast enhancement for improved Iris recognition", International conference on advanced computing technologies and applications, 2015.
[6] Dr.Sudeep Thepade, Jaya H. Dewan, "Image Compression Using Hybrid Wavelet Transform with Varying Proportions of Cosine, Haar, Walsh and Kekre Transforms with Assorted Color Spaces", 2014 International Conference on Control, Instrumentation, Communication and Computational Technologies (ICCICCT), 2014

[7] Dr. H. B. Kekre, Sudeep D. Thepade, Juhi Jain, Naman Agrawal, "Iris Recognition using Texture Features Extracted from Walshlet Pyramid", ACM-International Conference and Workshop on Emerging Trends in Technology (ICWET 2011). Thakur College of Engg. And Tech., Mumbai, 26-27 Feb 2011.

[8] Dr. Sudeep Thepade, Pushpa R. Mandal, Sachin Jadhav,"Performance Comparison of Novel Iris Recognition Techniques Using Partial Energies of Transformed Iris Images and Enegy CompactionWith Hybrid Wavelet Transforms", Annual IEEE India Conference (INDICON),2015

[9] Dr. H. B. Kekre, Dr. Tanuja K. Sarode, Sudeep D. Thepade and Ms. Sonal Shroff, "Instigation of Orthogonal Wavelet Transforms using walsh, Cosine, Hartley, Kekre Transforms and their use in Image Compression", (IJCSIS) International Journal of Computer Science and Information Security, Vol. 9, No. 6, 2011.

[10] PalackyUniversityIrisDatabase,http://www.advancesourc ecode.com/irisdatabase.asp.(Referred on 4 January 2016). 